\title{
Synchronous Early Gastric Cancer/Neuroendocrine Tumor Associated with Autoimmune Gastritis Completely Resected with Endoscopic Submucosal Dissection
}

\author{
Kimitoshi Kubo ${ }^{1}$, Noriko Kimura ${ }^{2}$, Soichiro Matsuda ${ }^{1}$, Katsuhiro Mabe ${ }^{1}$ and Mototsugu Kato ${ }^{1}$
}

\begin{abstract}
:
Synchronous early gastric cancer/neuroendocrine tumor (NET) associated with autoimmune gastritis is rare, and its endoscopic and pathological features remain poorly described. Screening esophagogastroduodenoscopy performed on a 71-year-old man revealed a whitish, superficial elevated lesion and a submucosal tumor with redness that appeared slightly centrally depressed. Endoscopic submucosal dissection (ESD) allowed these lesions to be resected with negative margins, and they were diagnosed as tubular adenocarcinoma, welldifferentiated type (tub1), pT1a (M) and NET G1, pT1b (SM). To our knowledge, this is the first report describing the endoscopic and pathological findings of synchronous early gastric cancer/NET that was amenable to complete resection with ESD.
\end{abstract}

Key words: gastric carcinoma, gastric NET, endoscopic treatment

(Intern Med 58: 2633-2637, 2019)

(DOI: 10.2169/internalmedicine.2679-19)

\section{Introduction}

In the 2010 WHO classification, gastrointestinal neuroendocrine tumors (NETs) are classified into NET G1, G2, and neuroendocrine carcinomas (NECs) according to the grade of malignancy of each component (1). While autoimmune gastritis is associated with two types of gastric neoplasms, i.e. gastric cancer and NET (2), synchronous gastric cancer/ NET associated with autoimmune gastritis is rare, and its endoscopic and pathological features remain poorly described.

We herein report a case of synchronous early gastric cancer/NET in a patient with autoimmune gastritis that was amenable to complete resection with endoscopic submucosal dissection (ESD).

\section{Case Report}

Screening esophagogastroduodenoscopy (EGD) performed on a 71-year-old man revealed a whitish, superficial elevated lesion and a submucosal tumor with redness that appeared slightly centrally depressed in the posterior wall of the gastric angle on white-light imaging (WLI) (Fig. 1A and B). On narrow-band imaging (NBI), the lesion was depicted as a whitish, well-circumscribed superficial lesion (Fig. 1C). Furthermore, an irregular microvascular pattern was shown to be present within the demarcation line on magnifying NBI (Fig. 1D). Based on these findings, the lesion was diagnosed as early gastric cancer.

An EGD biopsy revealed adenoma and endocrine cell micronests (ECMs). Laboratory data showed the patient to be positive for anti-parietal cells, anti-intrinsic factor antibodies, and hypergastrinemia (gastrin, up to $1,440 \mathrm{pg} / \mathrm{mL}$ ) but negative for serum Helicobacter pylori $\mathrm{IgG}$ antibody $(<3 \mathrm{U} / \mathrm{mL})$. In addition, an examination of the biopsy specimen revealed proximal-predominant gastric mucosal atrophy and scattered ECM in the proximal region (Fig. 2) with no evidence of $H$. pylori colonization, suggesting the presence of autoimmune gastritis.

ESD was performed on the suspected well-differentiated tubular adenocarcinoma/ NET for a biopsy and endoscopic

${ }^{1}$ Department of Gastroenterology, National Hospital Organization Hakodate National Hospital, Japan and ${ }^{2}$ Department of Pathology, National Hospital Organization Hakodate National Hospital, Japan

Received: January 15, 2019; Accepted: March 28, 2019; Advance Publication by J-STAGE: June 7, 2019

Correspondence to Dr. Kimitoshi Kubo, kubotti25@yahoo.co.jp 

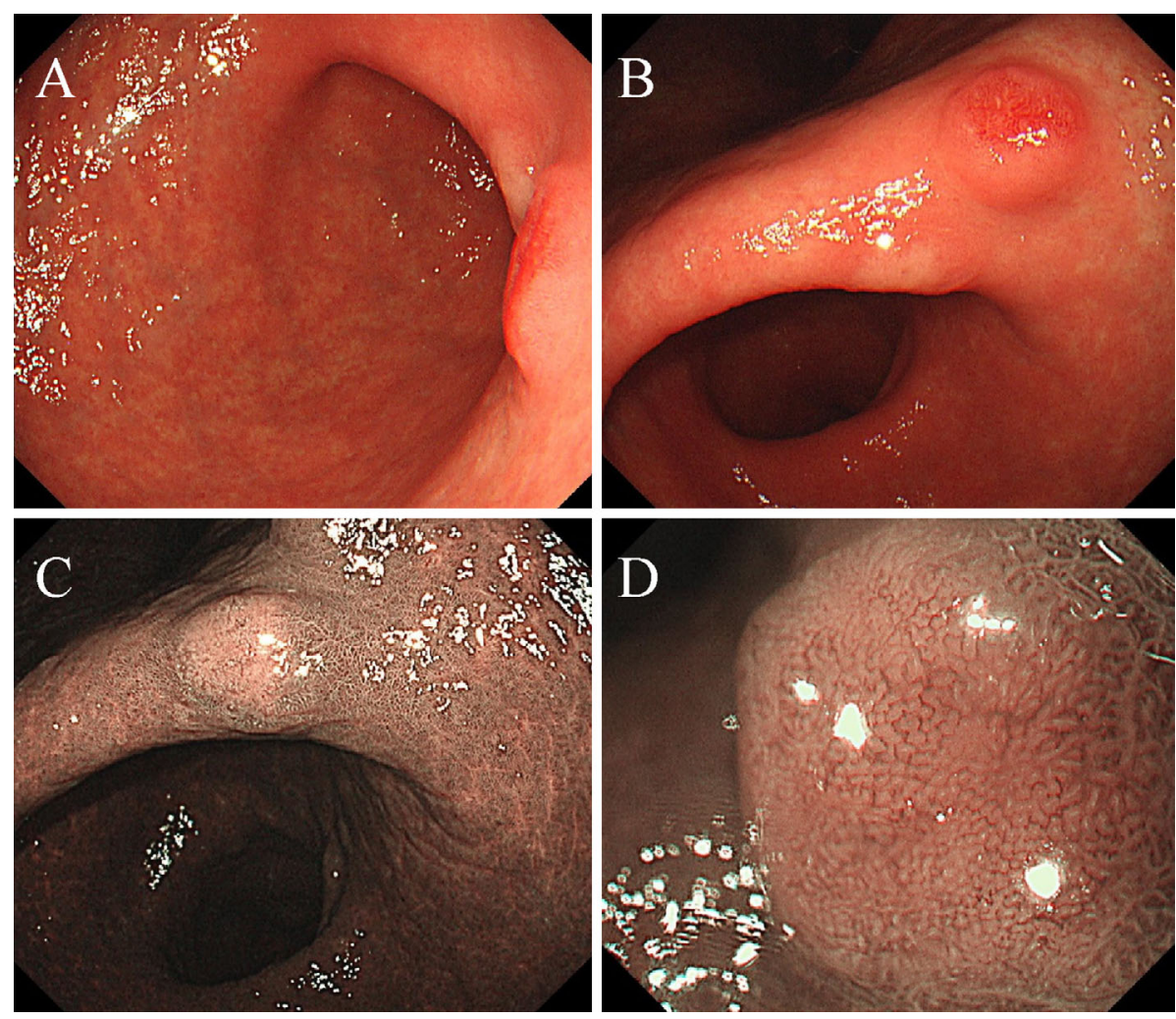

Figure 1. Esophagogastroduodenoscopy. A whitish, superficial elevated lesion and a submucosal tumor with redness that appeared slightly centrally depressed in the posterior wall of the gastric angle on WLI (A, B). A whitish, superficial elevated lesion was depicted as a whitish, well-circumscribed superficial lesion by NBI (C). An irregular microvascular pattern was shown to be present within the demarcation line on magnifying NBI (D).
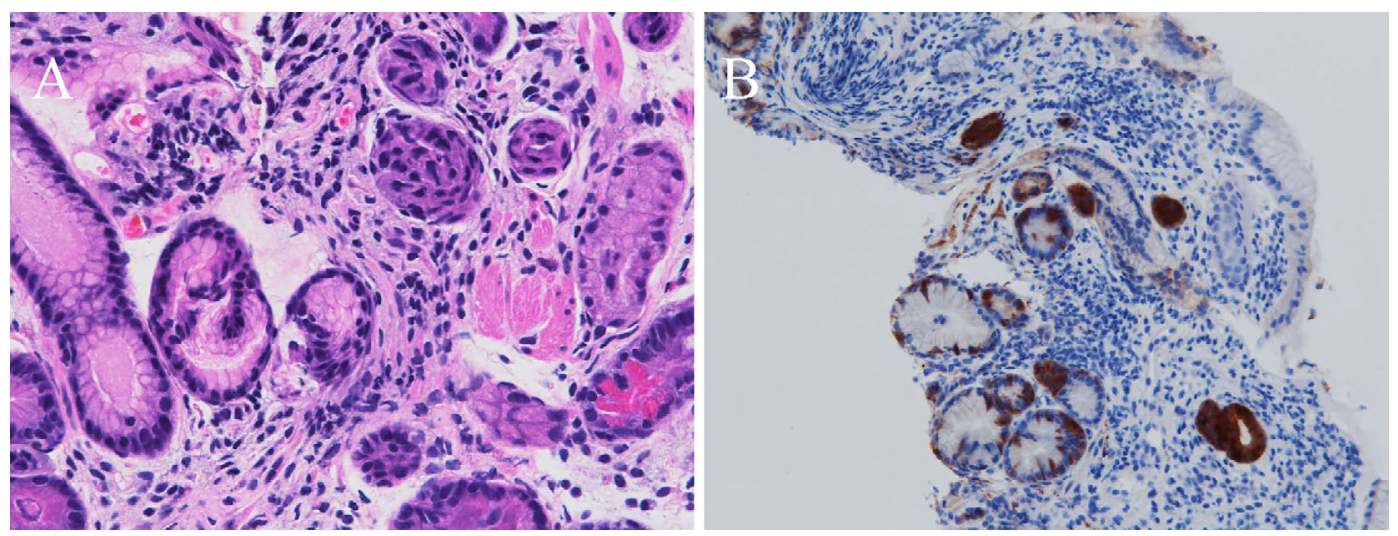

Figure 2. A histopathologic examination and immunohistological staining of the biopsy specimen from the gastric wall. (A) Endocrine cell micronests were detected in the background gastric mucosa (Hematoxylin and Eosin staining). (B) Endocrine cell micronests were shown to be positive for synaptophysin.

diagnosis. Macroscopically, the resected specimen was a 26x 18-mm lesion with a negative margin (Fig. 3). A histological examination showed the lesion to be well-differentiated type (tub1) tubular adenocarcinoma (Fig. 4A) and NET G1 with submucosal invasion (Fig. 4C), with the latter shown to be positive for chromogranin A (Fig. 4D) and synaptophysin. ECMs were detected in the deep mucosal layer of the tubular adenocarcinoma (Fig. 4B). The gastric lesion in the pa- tient was therefore diagnosed as synchronous 1) adenocarcinoma, type 0-IIa, measuring $6 \times 4 \mathrm{~mm}$, tub1, pT1a (M) and 2) NET, G1 measuring $8 \times 8 \mathrm{~mm}, \mathrm{pT} 1 \mathrm{~b}(\mathrm{SM})$. Each of these sections was shown to have negative margins. An EGD examination performed six months later revealed no signs of recurrence. In addition, a biopsy specimen revealed all other small NET-suspected lesions to be hyperplastic polyps. 


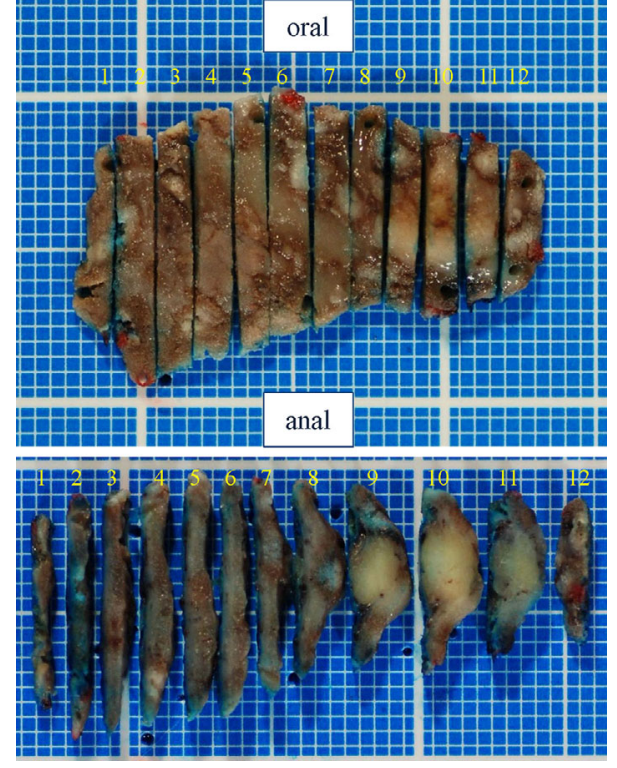

Figure 3. Macroscopic view of the resected specimen. A 0-IIa adenocarcinoma (tub1) measuring $6 \times 4 \mathrm{~mm}$ was shown to be present in sections $3-5$, and a submucosal tumor measuring $8 \times 8$ mm was shown to be present in sections 9-11.
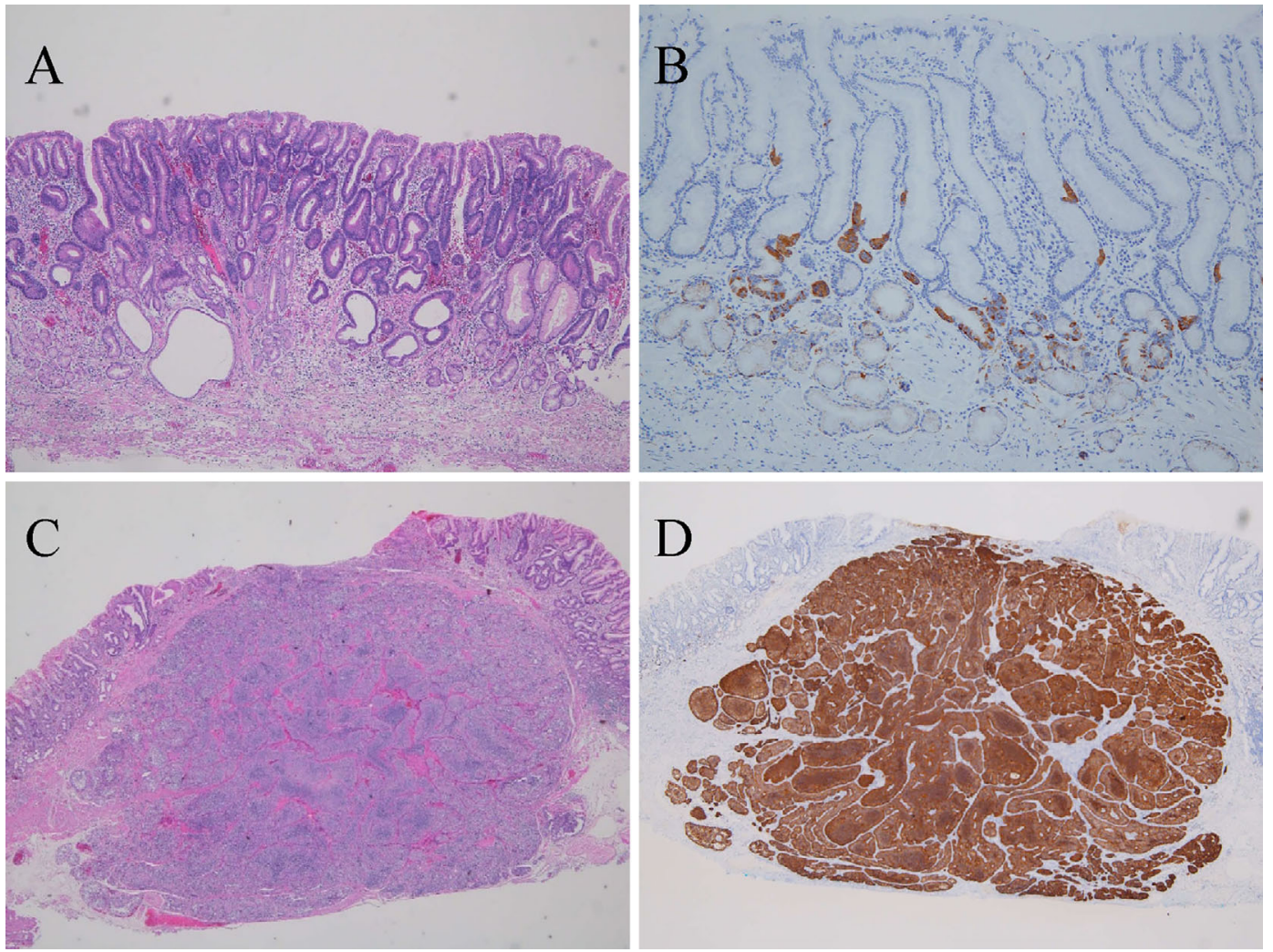

Figure 4. Findings of a histopathologic examination and immunohistological staining of the lesions in the posterior wall. (A) The histological examination showed well-differentiated type (tub1) tubular adenocarcinoma (Hematoxylin and Eosin staining). (B) Endocrine cell micronests positive for synaptophysin were detected in the deep mucosal layer of the tubular adenocarcinoma. (C) The histological examination showed NET G1 with submucosal invasion (C), which was positive for chromogranin A (D). 


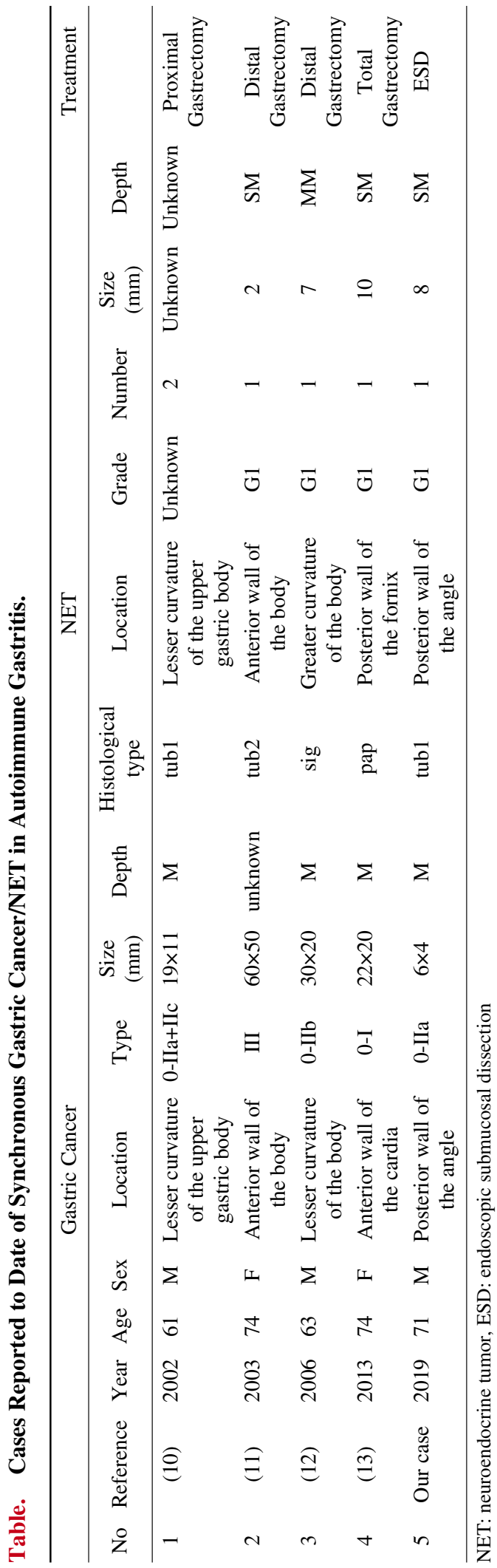

commonly employed endoscopic procedure $(6,7)$, ESD is reported to be associated with similar resection rates and complication rates $(8,9)$ and is a more effective modality than EMR for resecting NETs, which are often shown to have invaded the submucosa, as it allows the resection area to be checked during incision, despite greater technical difficulties (8). In the present case, we decided to perform ESD for the following reasons: 1) the lesions were small gastric NETs measuring $\leq 1 \mathrm{~cm}$; and 2) ESD would allow the two lesions to be resected in one attempt. Through ESD, the gastric cancer/NET lesion were completely resected with negative vertical margins. Thus far, four cases of synchronous gastric cancer/NET associated with autoimmune gastritis have been reported (Table) (10-13), all of which were diagnosed after total gastrectomy. To our knowledge, this is the first report describing synchronous early gastric cancer/ NET that was amenable to complete resection with ESD.

The second implication of our case is that the synchronous early gastric cancer/ ET was derived from the same atrophic mucosa associated with autoimmune gastritis. Gastric cancer and NET lesions are reportedly observed in 0.9$9 \%$ and $4-9 \%$ of patients with autoimmune gastritis, respectively (14). The pathogenesis of gastric cancer is attributed to atrophic gastritis resulting in intestinal metaplasia (2) or long-term hypergastrinemia (15), leading to the development of adenocarcinoma, while that of type I gastric NET is attributed to elevated gastrin secretion in response to the loss of negative feedback by parietal cells, wherein hypo-/ achlorhydria-induced hypergastrinemia results in enterochromaffin-like (ECL) cell hyperplasia and dysplasia, thus leading to type I gastric NET $(16,17)$. In our case, the patient was shown to be positive for anti-parietal cells, antiintrinsic factor antibodies, and hypergastrinemia (gastrin, up to $1,440 \mathrm{pg} / \mathrm{mL}$ ) but negative for serum $H$. pylori $\mathrm{IgG}$ antibody. Again, given that scattered ECMs were recognized in the background gastric mucosa and that no continuity was shown between the gastric cancer lesion and the NET, it was concluded that the synchronous early gastric cancer lesion/ NET had been derived separately from the same atrophic mucosa associated with autoimmune gastritis.

In conclusion, early gastric cancer/NET G1 may present as a synchronous lesion derived from autoimmune gastritis. Patients with autoimmune gastritis require endoscopic surveillance for potential gastric cancer/NET.

The authors state that they have no Conflict of Interest (COI).

\section{References}

1. Bosman FT, Carneiro F, Hruban RH, et al. WHO classification of tumours of the digestive system. 4th ed. Lyon, IARC, 2010.

2. Bizzaro N, Antico A, Villalta D. Autoimmunity and gastric cancer. Int J Mol Sci 19: 377, 2018.

3. Delle Fave G, Kwekkeboom DJ, Van Cutsem E, et al. ENETS consensus guidelines for the management of patients with gastroduodenal neoplasms. Neuroendocrinology 95: 74-87, 2012.

4. Kulke MH, Shah MH, Benson AB III, et al. Neuroendocrine tumors, version 1.2015. J Natl Compr Canc Netw 13: 78-108, 2015.

5. Scherübl H, Cadiot G. Early gastroenteropancreatic neuroendocrine tumors: endoscopic therapy and surveillance. Visc Med 33: 332-338, 2017.

6. Ichikawa J, Tanabe S, Koizumi W, et al. Endoscopic mucosal resection in the management of gastric carcinoid tumors. Endoscopy 35: 203-206, 2003.

7. Merola E, Sbrozzi-Vanni A, Panzuto F, et al. TypeIgastric carci- 
noids: a prospective study on endoscopic management and recurrence rate. Neuroendocrinology 95: 207-213, 2012.

8. Li QL, Zhang YQ, Chen WF, et al. Endoscopic submucosal dissection for foregut neuroendocrine tumors: an initial study. World J Gastroenterol 18: 5799-5806, 2012.

9. Kim HH, Kim GH, Kim JH, et al. The efficacy of endoscopic submucosal dissection of type I gastric carcinoid tumors compared with conventional endoscopic mucosal resection. Gastroenterol Res Pract 2014: 253860, 2014.

10. Hiroyoshi M, Ogino K, Moritomo $\mathrm{H}$, et al. A case of gastric carcinoids associated with type A gastritis and gastric cancer. Nihon Shokakibyo Gakkai Zasshi 99: 270-274, 2002 (in Japanese).

11. Takahashi H, Koike J. Double cancer of stomach, adenocarcinoma and carcinoid tumor with type A gastritis: a case report. Jpn J Diagn Pathol 20: 124-127, 2003 (in Japanese, Abstract in English).

12. Yang L, Zhang HT, Zhang X, et al. Synchronous occurrence of carcinoid, signet-ring cell carcinoma and hepaterotopic pancreatic tissue in stomach: a case report and literature review. World J Gastroenterol 28: 7216-7220, 2006.

13. Fujisawa T, Takata M, Nishizawa A, et al. Combination of early gastric carcinoma and multiple carcinoids accompanied by type A gastritis, report of a case. Stomach and Intestine 48: 1799-1809, 2013 (in Japanese, Abstract in English).

14. De Block CE, De Leeuw IH, Van Gaal LF. Autoimmune gastritis in type 1 diabetes: a clinically oriented review. J Clin Endocrinol Metab 93: 363-371, 2008.

15. Lahner E, Esposito G, Pilozzi E, et al. Gastric cancer in patients with type I gastric carcinoids. Gastric Cancer 18: 564-570, 2015.

16. Burkitt MD, Pritchard DM. Review article: pathogenesis and management of gastric carcinoid tumours. Aliment Pharmacol Ther 24: 1305-1320, 2006.

17. Minalyan A, Benhammou NJ, Artashesyan A, et al. Autoimmune gastritis: current perspectives. Clin Exp Gastroenterol 1: 19-27, 2017.

The Internal Medicine is an Open Access journal distributed under the Creative Commons Attribution-NonCommercial-NoDerivatives 4.0 International License. To view the details of this license, please visit (https://creativecommons.org/licenses/ by-nc-nd/4.0/).

(C) 2019 The Japanese Society of Internal Medicine

Intern Med 58: 2633-2637, 2019 